\title{
Video Transcoder in DCT-Domain Spatial Resolution Reduction Using Low-Complexity Motion Vector Refinement Algorithm
}

\author{
Tsung-Han Tsai, Yu-Fun Lin, and Hsueh-Yi Lin \\ Department of Electrical Engineering, National Central University, Jhongli, Taoyuan County 32001, Taiwan
}

Correspondence should be addressed to Hsueh-Yi Lin, davidlin409@dsp.ee.ncu.edu.tw

Received 26 February 2008; Revised 30 June 2008; Accepted 2 September 2008

Recommended by Moon Kang

\begin{abstract}
We address the topic of spatial-downscaling video transcoder in DCT-domain. The proposed techniques include the hierarchical fast motion resampling (HFMR) with accuracy motion resampling, the fast refinement for nonintegral (FRNI) motion vector $(\mathrm{MV})$, and the dynamic regulating search (DRS) with low-complexity motion vector refinement. Two kinds of motion vector refinement algorithms in DRS are designed for different architectures and applications. Based on brute-force motion compensation in DCT-domain (MC-DCT), FRNI can provide better quality than nonrefine MV and reduce the complexity. DRS can utilize the filter for half-pixel MV in MC-DCT and it is an efficient method for extracting MC-DCT block to improve the performance further. From the experiments, the proposed algorithms can improve the entire quality and also reduce the complexity for DCTdomain video transcoder.
\end{abstract}

Copyright (C) 2008 Tsung-Han Tsai et al. This is an open access article distributed under the Creative Commons Attribution License, which permits unrestricted use, distribution, and reproduction in any medium, provided the original work is properly cited.

\section{INTRODUCTION}

Recently, the masses have brought up all kinds of multimedia services to more and more demands on digital video, such as video on demand, distance learning, and video surveillance. In these applications, compressed digital video is a major component of the multimedia data. Video coding technology makes those applications feasible. In video coding, an analog video signal is digitized and then compressed to fit into desirable bandwidth.

In the context of coding and transmission, there is an increasing need to perform many types of conversions to accommodate terminal constraints, network limitations, or preferences of user. There exists a rich set of contents that are rapidly growing by the day. On the other hand, we have terminals with varying capabilities. Connection between the terminals requires conversions to adapt between heterogeneous network configurations and the differences between terminal constraints. Among existing techniques, video transcoding has allowed user to convert a previously compressed bit-stream into another format to meet various multimedia services. The relative work is a process of converting a previously compressed video bit-stream into another bit-stream with a lower bitrate, a different resolution (e.g., downscaling), a different coding format (e.g., the conversion between MPEG-x and H.26x, or adding error resilience), and so forth.

H.264 and H.263 play different roles in current industry. H.264 is computationally expensive in software. Hardware realization can enhance the computation speed to achieve real-time goal. However, the real-time requirement on software is hard to achieve in H.264. If simple methods are applied, resulting performance might not be satisfactory. When real-time software is desired without considering too much performance gain, H.263 becomes an alternative. Especially in the age with wider Internet capacity, occupying larger bandwidth might be acceptable. Nowadays, most of existing mobile phones are capable of watching movies from 3GPP or MV4 format. Video clips from the phoneembedded camera are stored in 3GPP format. According to the general description, 3GPP is version of H.263 for streaming and mobile applications. Since it is widely adopted in mobile devices, transcoding between mobile phone can be conducted by H.263 transcoding.

Video transcoding has allowed users to convert a previously compressed bit-stream into another format to meet 


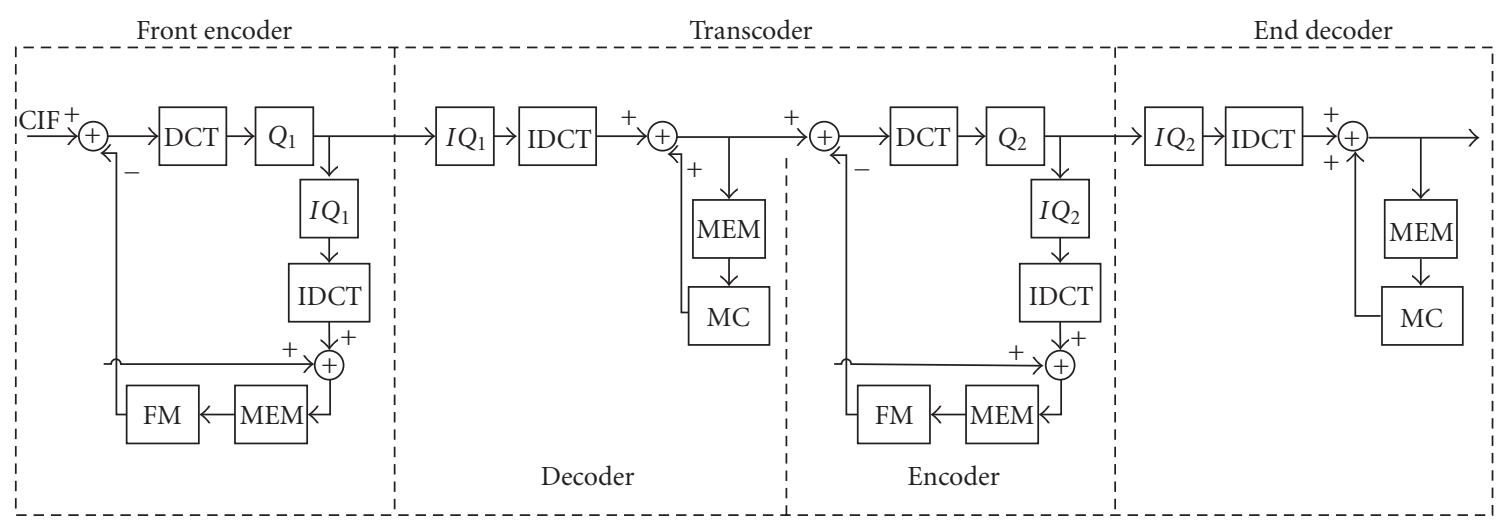

FIGURE 1: A straightforward realization of video transcoder.

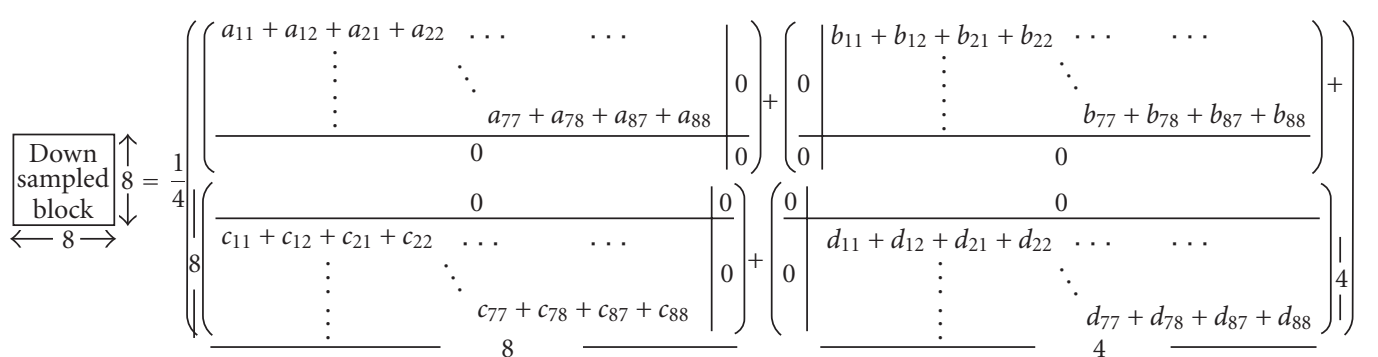

Figure 2: Pixel averaging and down-sampling performed on $8 \times 8$ block basis.

various multimedia services. It is classified into three categories: spatial transcoding, temporal transcoding, and special application transcoding. Among those considerations, the display resolution of terminal constraint is especially important [1-7]. Therefore, we focus our attention on the problem of reduced resolution transcoding. Specifically, we consider techniques and architectures to convert a compressed video bit-stream with one spatial resolution to the output with half of original spatial resolution.

A straightforward realization of video transcoder is to cascade a decoder followed by an encoder directly, as shown in Figure 1. However, the computational complexity is timeconsuming so that it is not suitable for real-time applications. To avoid drift error [2] and reduce computational complexity, the close loop DCT-domain architecture becomes the main stream in video transcoding. The DCT-domain approach reduces computational complexity by $40 \%$ than the pixel-domain one, meanwhile preserving comparable picture quality with little degradation [3]. To construct the DCT-domain architecture, different approaches are applied, such as those in [5-7].

In this article, the novel video transcoder method in DCT-domain spatial resolution reduction is proposed. It includes the fast motion resampling method, called Hierarchical fast motion resampling (HFMR), and two motion vector refinement (MVR) algorithms, called fast refinement for nonintegral (FRNI) motion vector and dynamic regulation search (DRS). Based on brute-force motion compensation in DCT-domain (MC-DCT), FRNI can provide better quality than nonrefine motion vector and reduces the complexity. In DRS, it utilizes the filter for half-pixel motion vector in
MC-DCT [8] and efficient method for extracting MC-DCT block $[9,10]$ to further improve the performance. Two kinds of MVR algorithms in DRS are further designed for different architectures. This paper is organized as follows: in Section 2, a brief introduction and related works are introduced; in Section 3, our proposed algorithms are proposed; in Section 4, we show our experimental result for all proposed algorithms; and finally, our concluding remarks are given in Section 5.

\section{FUNDAMENTALS IN DCT-DOMAIN VIDEO TRANSCODING}

\subsection{DCT-domain down-conversion}

In pixel-domain transcoder, the downscaled video is composed of summation of four pixels into a new one. When DCT is removed from original coding flow, modification is necessary to achieve the same functionality. The framework of extending the simple pixel averaging and down-sampling to the DCT-domain was introduced by Chang and Messerschmitt [10] and subsequently optimized for fast processing by Merhav, Bhaskaran [11], and Merhav [12]. In DCTdomain down-sampling, pixel averaging and down-sampling are performed with the smallest unit of $8 \times 8$ block of pixels, as shown in Figure 2. The $a, b, c, d$ in the figure indicate four neighboring blocks, respectively. Afterward, all matrices are transformed into DCT-domain.

Downscaling is separated into three stages. At the first stage, four adjacent pixels in $b_{i}$ are summed up to create a new pixel. This implies that the input block is replaced by 


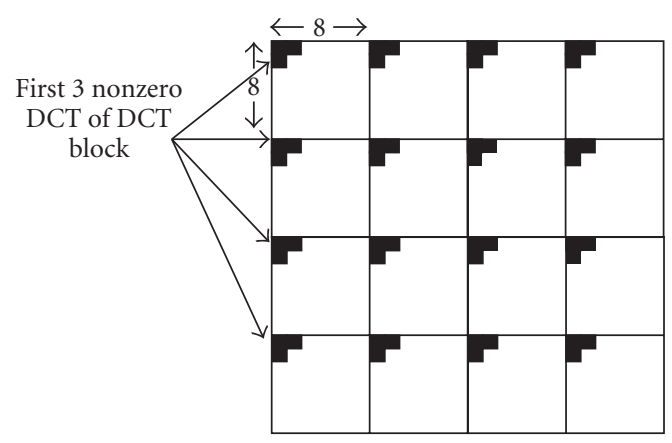

FIgURE 3: WLF-max motion vector composition algorithm.

$4 \times 4$ pixels in the top left corner; and the rest of the block is padded with zeros. At the second stage, the adjacent blocks are shifted according to the location of the underlying block $b_{i}$. That is, the $4 \times 4$ pixels are shifted to the top left corner in $b_{1}$ and to the top right in $b_{2}$ and so forth. Finally, the four new blocks are added and divided by four to generate the down-sampled block $b$. These steps are formulated by (1). In the figure, $Q_{1}$ and $Q_{2}$ are defined by (2) and (3). However, $Q_{1}^{\prime}$ and $Q_{2}^{\prime}$ indicate the transpose of $Q_{1}$ and $Q_{2}$, while $q_{1}$ and $q_{2}$ are defined by two matrices, as formulated in (4) and (5):

$$
\begin{gathered}
B=\frac{1}{4}\left(Q_{1} B_{1} Q_{1}^{\prime}+Q_{2} B_{2} Q_{2}^{\prime}+Q_{3} B_{3} Q_{3}^{\prime}+Q_{4} B_{4} Q_{4}^{\prime}\right), \\
Q_{1}=\operatorname{DCT}\left(q_{1}\right) \\
Q_{2}=\operatorname{DCT}\left(q_{2}\right) \\
q_{1}=\left[\begin{array}{llllllll}
1 & 1 & 0 & 0 & 0 & 0 & 0 & 0 \\
0 & 0 & 1 & 1 & 0 & 0 & 0 & 0 \\
0 & 0 & 0 & 0 & 1 & 1 & 0 & 0 \\
0 & 0 & 0 & 0 & 0 & 0 & 1 & 1 \\
0 & 0 & 0 & 0 & 0 & 0 & 0 & 0 \\
0 & 0 & 0 & 0 & 0 & 0 & 0 & 0 \\
0 & 0 & 0 & 0 & 0 & 0 & 0 & 0 \\
0 & 0 & 0 & 0 & 0 & 0 & 0 & 0
\end{array}\right] \\
q_{2}=\left[\begin{array}{llllllll}
0 & 0 & 0 & 0 & 0 & 0 & 0 & 0 \\
0 & 0 & 0 & 0 & 0 & 0 & 0 & 0 \\
0 & 0 & 0 & 0 & 0 & 0 & 0 & 0 \\
0 & 0 & 0 & 0 & 0 & 0 & 0 & 0 \\
1 & 1 & 0 & 0 & 0 & 0 & 0 & 0 \\
0 & 0 & 1 & 1 & 0 & 0 & 0 & 0 \\
0 & 0 & 0 & 0 & 1 & 1 & 0 & 0 \\
0 & 0 & 0 & 0 & 0 & 0 & 1 & 1
\end{array}\right]
\end{gathered}
$$

Owing to the unitary property of the DCT, (1) is performed in the DCT-domain by simply transforming all participating blocks to the transform-domain. This method is hereby referred to as pixel averaging and down-sampling.

\subsection{Fast motion resampling (FMR)}

Fast motion resampling involves generation of new motion vector from existing 4 blocks. When the predicted motion vectors are accurate, less refinement points are searched. Lots of algorithms are designed for accurate approximation. Brief descriptions of the algorithms are described as follows.

(1) The average. This is the most straight-forward approach by averaging existing motion vectors, which is formulated by (6):

$$
\begin{aligned}
& V(x)=\frac{1}{2} \times\left(\frac{1}{4} \sum_{i=1}^{4} V_{i}(x)\right), \\
& V(y)=\frac{1}{2} \times\left(\frac{1}{4} \sum_{i=1}^{4} V_{i}(y)\right) .
\end{aligned}
$$

(2) The median. Let $V$ be the set of four adjacent motion vectors $\left(v_{1}, v_{2}, v_{3}, v_{4}\right)$. The algorithm is to calculate the sum of the mutual distances, as described in:

$$
v=\frac{1}{2} \arg \left(\min _{V \in\left\{v_{1}, v_{2}, v_{3}, v_{4}\right\}}\left(\sum_{j=1, j \neq i}^{4}\left\|v_{i}-v_{j}\right\|\right)\right) .
$$

(3) WLF-MAX. The objective is to estimate optimal motion vector with reduced complexity. Visual quality matrix (VQM) [13], as described in Table 1, is utilized to weight individual AC coefficients. To further reduce the complexity, adopted coefficients are reduced from 64 to first three nonzero coefficients with zigzag scan order. The algorithm is formulated according to:

$$
\begin{gathered}
\operatorname{ACT}_{i}=\sum_{k=1}^{4} \operatorname{Abs}(\operatorname{DCT}(m, n)) \times \operatorname{VQM}(m, n), \\
i, m, n=1,2,3,4, \\
v=\frac{1}{2} \mathrm{mv}_{i} \| \mathrm{ACT}_{i} \text { is maximum, }
\end{gathered}
$$

where " $v$ " is the composed motion vector for downsized video, while " $\mathrm{mv}_{i}$ " denotes the motion vector of block $i$ in a macroblock, and $\operatorname{DCT}(m, n)$ denotes the DCT coefficient in the $m$ th row and $n$th column, relative to VQM matrix. Overall process is illustrated in Figure 3.

(4) ACT-weighted. In this method, the distance between each vector and the rest is calculated as sum of the activity-weighted distances by (9). The activity is the squared or absolute sum of DCT coefficients, the number of nonzero DCT coefficients, or simply the DC value. The work in [14] adopts the squared sum to measure the activity. Optimal motion vector is obtained with the least distance from all

$$
d_{i}=\frac{1}{\mathrm{ACT}} \sum_{j=1, j \neq i}^{4}\left\|v_{i}-v_{j}\right\| .
$$

For composing the new motion vectors to the downsized version, the four techniques were compared in [14]. It turns out that the ACT-weighted scheme outperforms the other techniques. 
TABLE 1: Visual quantization matrix for images.

\begin{tabular}{lcccccccc}
\hline Row/Col & 1 & 2 & 3 & 4 & 5 & 6 & 7 \\
\hline 1 & 1 & 0.9962 & 0.985 & 0.9668 & 0.9423 & 0.9122 & 0.8775 \\
2 & 0.9944 & 0.9906 & 0.9795 & 0.9615 & 0.9372 & 0.9073 & 0.873 \\
3 & 0.9778 & 0.9741 & 0.9633 & 0.9458 & 0.9221 & 0.8931 & 0.8596 & 0.8351 \\
4 & 0.9511 & 0.9476 & 0.9373 & 0.9206 & 0.8979 & 0.8701 & 0.8381 & 0.8026 \\
5 & 0.9158 & 0.9125 & 0.9028 & 0.8871 & 0.8658 & 0.8396 & 0.8094 & 0.7759 \\
6 & 0.8734 & 0.8704 & 0.8615 & 0.8469 & 0.8272 & 0.8029 & 0.7748 & 0.7437 \\
7 & 0.826 & 0.8232 & 0.8151 & 0.8018 & 0.7837 & 0.7615 & 0.7358 & 0.7072 \\
8 & 0.7752 & 0.7727 & 0.7654 & 0.7534 & 0.7371 & 0.7171 & 0.6937 & 0.6678 \\
\hline
\end{tabular}

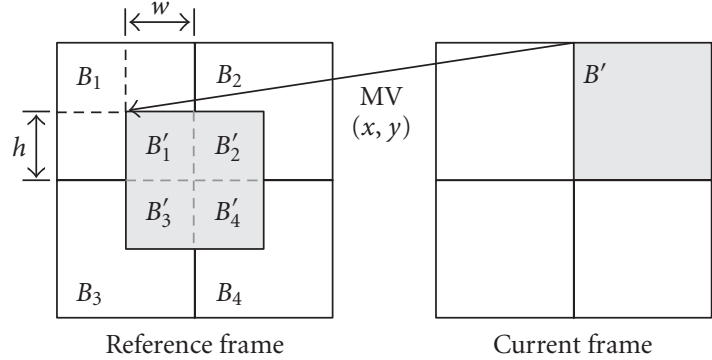

FIGURE 4: DCT-domain motion compensation.

\subsection{DCT-domain motion compensation (MC-DCT)}

MC-DCT is the process to manipulate motion compensation in DCT-domain approaches. From Figure 4, a motion vector specified as $(x, y)$ means that the block $B^{\prime}$ is predicted from the reference block with corresponding displacement. The reference block occupies four blocks in the previous frame. Thus, proper manipulation is applied to obtain the prediction/compensation content $B$.

It is separated into three steps, as illustrated in Figure 5. The first step is to extract corresponding region from reference blocks. The second is to shift the pixels to respective locations. Finally, the results are summed up to obtain the prediction content. According to the shift distance, corresponding matrices are applied $\left(H_{i 1}\right.$ and $\left.H_{i 2}\right)$ to obtain the final result. $H_{i 1}$ is used for horizontal translation, and $H_{i 2}$ is used for vertical translation. Since four blocks are involved in composing single prediction block, four matrices, as described in Table 2, are obtained. However, $I_{w}$ and $I_{h}$ are identity matrices with size $w \times w$ and $h \times h$, respectively, while $h$ is number of rows extracted, and $w$ is the number of rows extracted.

DCT operation means to remove DCT with proper modifications. According to the derivation in (10), the matrices $H_{i 1}$ and $H_{i 2}$ are transformed and stored first:

$$
\begin{aligned}
\operatorname{DCT}\left(B_{i}^{\prime}\right) & =\operatorname{DCT}\left(H_{i 1} \cdot B_{i} \cdot H_{i 2}\right) \\
& =\operatorname{DCT}\left(H_{i 1}\right) \operatorname{DCT}\left(B_{i}\right) \operatorname{DCT}\left(H_{i 2}\right) .
\end{aligned}
$$

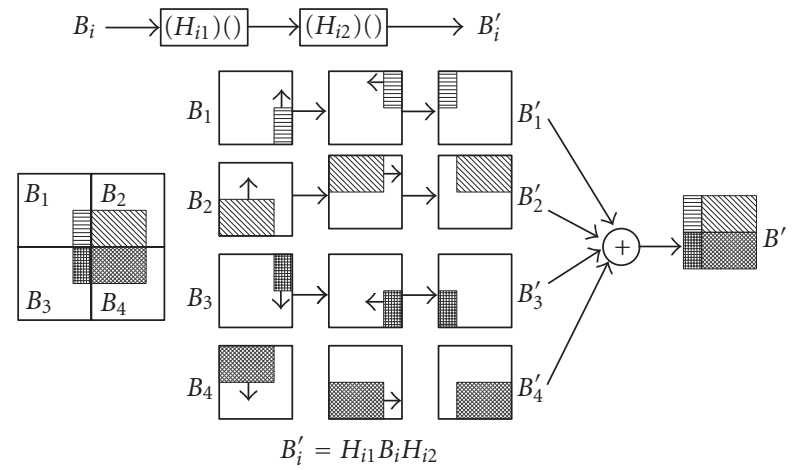

FIGURE 5: A new image block, $B^{\prime}$, consisting of contributions $\left(B_{1}^{\prime}\right.$, $B_{2}^{\prime}, B_{3}^{\prime}$, and $\left.B_{4}^{\prime}\right)$ from four original neighboring blocks $\left(B_{1}, B_{2}, B_{3}\right.$, and $B_{4}$ ).

TABLe 2: Matrices of $H_{i 1}$ and $H_{i 2}$.

\begin{tabular}{llcc}
\hline Subblock & Position & $H_{i 1}$ & $H_{i 2}$ \\
\hline$B_{1}$ & Low right & $\left(\begin{array}{cc}0 & I_{h 1} \\
0 & 0\end{array}\right)$ & $\left(\begin{array}{cc}0 & 0 \\
I_{w 1} & 0\end{array}\right)$ \\
$B_{2}$ & Lower left & $\left(\begin{array}{cc}0 & I_{h 2} \\
0 & 0\end{array}\right)$ & $\left(\begin{array}{cc}0 & I_{w 2} \\
0 & 0\end{array}\right)$ \\
$B_{3}$ & Upper right & $\left(\begin{array}{cc}0 & 0 \\
I_{h 3} & 0\end{array}\right)$ & $\left(\begin{array}{cc}0 & 0 \\
I_{w 3} & 0\end{array}\right)$ \\
$B_{4}$ & Upper left & $\left(\begin{array}{cc}0 & 0 \\
I_{h 4} & 0\end{array}\right)$ & $\left(\begin{array}{cc}0 & I_{w 4} \\
0 & 0\end{array}\right)$ \\
\hline
\end{tabular}

Afterward, simple matrix multiplication is applied to allocate part of predicted coefficients. Since $B^{\prime}$ is the summation of $B_{i}^{\prime}$, overall prediction coefficients are formulated in:

$$
\begin{aligned}
\operatorname{DCT}\left(B^{\prime}\right) & =\operatorname{DCT}\left(B_{1}^{\prime}+B_{2}^{\prime}+B_{3}^{\prime}+B_{4}^{\prime}\right)=\sum_{i=1}^{4} \operatorname{DCT}\left(B_{i}^{\prime}\right) \\
& =\sum_{i=1}^{4} \operatorname{DCT}\left(H_{i 1}\right) \operatorname{DCT}\left(B_{i}\right) \operatorname{DCT}\left(H_{i 2}\right) .
\end{aligned}
$$

The block numbering order is shown in Figure 4.

Since matrix multiplications are required for prediction, efficient search algorithm becomes a key during refinement. 


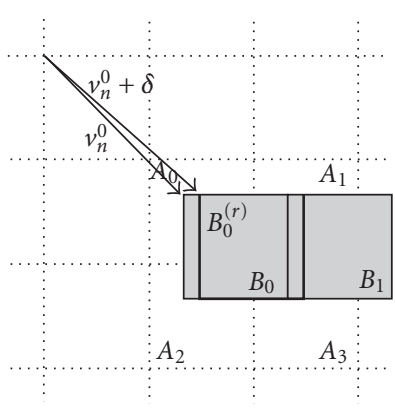

(a)

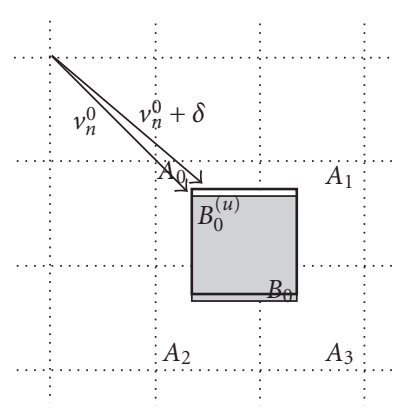

(c)

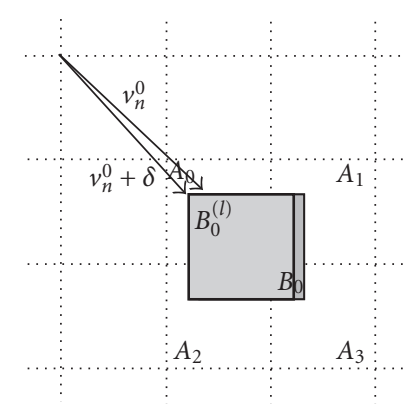

(b)

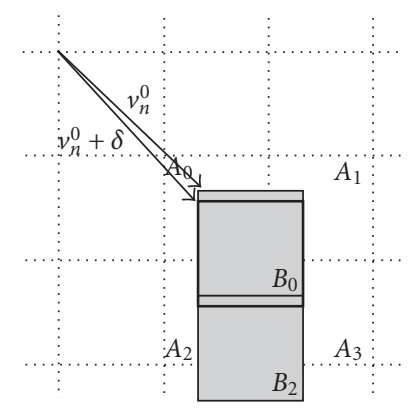

(d)
FIGURE 6: Overlap property of MC block predicted by $+\delta$, where $\delta=$ $\{(1,0),(-1,0),(0,1),(0,-1)\}$. (a) Overlap property of $B_{0}$ predicted by $v_{n}^{0}+(1,0)$. (b) Overlap property of $B_{0}$ predicted by $v_{n}^{0}+(-1,0)$. (c) Overlap property of $B_{0}$ predicted by $v_{n}^{0}+(0,1)$. (d) Overlap property of $B_{0}$ predicted by $v_{n}^{0}+(0,-1)$.

\subsection{Efficient method for extracting MC-DCT block in MVR}

To apply the criterion function of (17), the motion compensated DCT macroblock with motion vector of $\left(v_{n}^{0}+\delta\right)$ is to be extracted from the reference frame. Let the MCDCT macroblock $\widehat{M}_{n}$ (predicted by $v_{n}^{0}$ of $\widehat{\Omega}_{n}$ ) be composed of four blocks $\hat{B}_{0}, \widehat{B}_{1}, \widehat{B}_{2}$, and $\widehat{B}_{3}$ according to relative locations (top left, top right, bottom left, bottom right), respectively. The extraction is to find the MC-DCT block from the intersecting blocks $A_{i}(i=0,1,2,3)$ pointed by a motion vector $(\mathrm{MV})$ in the reference frame $\hat{f}_{k-1}$. One possible solution is illustrated in (11). However, it is too heavy for real-time application.

The work in [9] exploited the overlapping property of consecutive prediction. As shown in Figure 6(a), the MCDCT block, predicted by $v_{n}^{0}+(1,0)$, is displaced from $B_{0}$ by one pixel in the right. The superscript " $r$ " in $\widehat{B}_{0}^{r}$ denotes the right displacement by one pixel. (Similarly, the superscripts "l," " $u$," and " $d "$ denote the left, upward, and downward displacement by one pixel, resp.) Thus, $\hat{B}_{0}^{r}$ overlaps $B_{0}$ by 8 $\times 7$ pixels and $B_{1}$ by $8 \times 1$ pixels (Figure $6(\mathrm{a})$ ). To extract $\widehat{B}_{0}^{r}$, (13) is proposed, where

$$
R=\left(\begin{array}{cc}
0 & 0 \\
I_{7} & 0
\end{array}\right), \quad S=\left(\begin{array}{cc}
0 & I_{1} \\
0 & 0
\end{array}\right)
$$

$I_{k}$ is an identity matrix with size $k \times k$ :

$$
\begin{gathered}
W_{r}=\left(\begin{array}{cc}
0 & T_{m x} \\
0 & 0
\end{array}\right), \\
\hat{B}_{0}^{r}=\widehat{B}_{0} \hat{R}+\widehat{B}_{1} \widehat{S}, \\
\widehat{B}_{1}^{r}=\widehat{B}_{1} \hat{R}+\sum_{i=1,3} \hat{P}_{i} \widehat{A}_{i} \widehat{W}_{r}, \\
\hat{B}_{2}^{r}=\widehat{B}_{2} \hat{R}+\widehat{B}_{3} \widehat{S}, \\
\hat{B}_{3}^{r}=\widehat{B}_{3} \hat{R}+\sum_{j=1,3} \hat{P}_{j} \widehat{A}_{j} \widehat{W}_{r} .
\end{gathered}
$$

For the case $\hat{B}_{0}^{l}$ predicted by $v_{n}^{0}+(-1,0), \hat{B}_{0}^{l}$ overlaps $B_{0}$ by 8 $\times 7$ pixels and partially overlaps $A_{0}$ and $A_{2}$ (Figure 6(b)). To extract $\widehat{B}_{0}^{l}$, [9] proposes (14), where $\hat{R}^{T}$ is $\operatorname{DCT}\left(R^{T}\right)$,

$$
\begin{gathered}
W_{l}=\left(\begin{array}{rr}
0 & 0 \\
U_{8-m x} & 0
\end{array}\right), \\
\hat{B}_{0}^{l}=\widehat{B}_{0} \hat{R}^{T}+\sum_{i=0,2} \hat{P}_{i} \widehat{A}_{i} \widehat{W}_{l}, \\
\hat{B}_{1}^{l}=\widehat{B}_{1} \hat{R}^{T}+\widehat{B}_{0} \widehat{S}^{T}, \\
\hat{B}_{2}^{l}=\hat{B}_{2} \hat{R}^{T}+\sum_{j=0,2} \hat{P}_{j} \hat{A}_{j} \widehat{W}_{l}, \\
\hat{B}_{3}^{l}=\hat{B}_{3} \hat{R}^{T}+\widehat{B}_{2} \hat{S}^{T} .
\end{gathered}
$$

$U_{k}$ is a matrix with size $k \times k$, where only $(0,0)$ th component is 1 with others being zero. Similarly, [9] propose (15) and (16) to extract $\widehat{B}_{0}^{u}$ (predicted by $\left.v_{n}^{0}+(0,1)\right)$ and $\hat{B}_{0}^{d}$ (predicted by $\left.v_{n}^{0}+(0,-1)\right)$, respectively,

$$
\begin{aligned}
& W_{u}=\left(\begin{array}{cc}
0 & U_{8-m y} \\
0 & 0
\end{array}\right), \\
& \widehat{B}_{0}^{u}=\widehat{R} \widehat{B}_{0}+\sum_{i=0,1} \widehat{W}_{u} \widehat{A}_{i} \widehat{Q}_{i}, \\
& \widehat{B}_{1}^{u}=\widehat{R} \widehat{B}_{1}+\sum_{j=0,1} \widehat{W}_{u} \widehat{A}_{j} \widehat{Q}_{j}, \\
& \widehat{B}_{2}^{u}=\widehat{R} \widehat{B}_{2}+\widehat{S} \widehat{B}_{0}, \\
& \hat{B}_{3}^{u}=\hat{R} \widehat{B}_{3}+\widehat{S} \widehat{B}_{1}, \\
& W_{d}=\left(\begin{array}{cc}
0 & 0 \\
T_{m y} & 0
\end{array}\right), \\
& \hat{B}_{0}^{d}=\hat{R}^{T} \widehat{B}_{0}+\hat{S}^{T} \widehat{B}_{2}, \\
& \hat{B}_{1}^{d}=\hat{R}^{T} \widehat{B}_{1}+\hat{S}^{T} \widehat{B}_{3}, \\
& \widehat{B}_{2}^{d}=\widehat{R}^{T} \widehat{B}_{2}+\sum_{i=2,3} \widehat{W}_{d} \hat{A}_{i} \hat{Q}_{i}, \\
& \widehat{B}_{3}^{d}=\widehat{R}^{T} \widehat{B}_{3}+\sum_{j=2,3} \widehat{W}_{d} \widehat{A}_{j} \hat{Q}_{j} .
\end{aligned}
$$

For the case $\hat{B}_{1}^{\text {dir }}$, where $\operatorname{dir} \in\{r, l, u, d\}$, a similar algorithm is applied to extract the DCT block predicted by $v_{n}^{0}+\delta$ using 


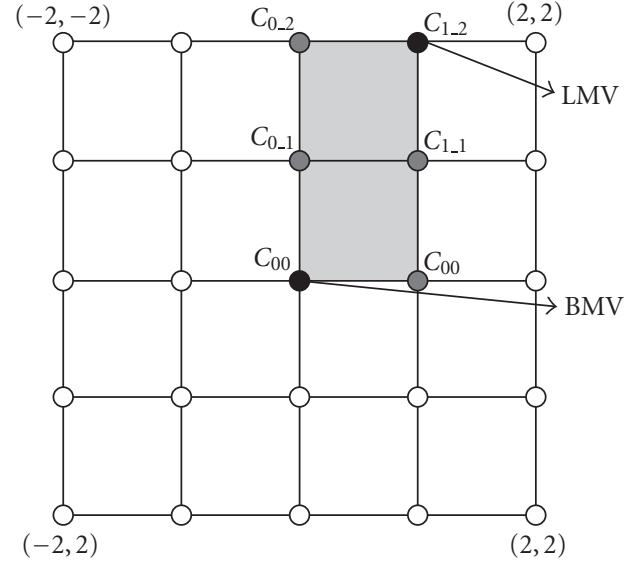

FIGURE 7: Fast searching pattern using LMV.

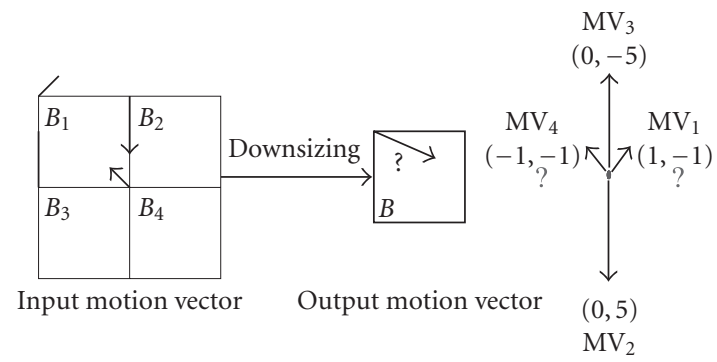

FIgURE 8: Example of the different direction motion vectors having the same value.

the overlap information. If the four intersected blocks are denoted as $A_{i}(i \in\{0,1,2,3\})$, the required equations for extracting the desired DCT block are the second equations of (13), (14), (15), and (16). $T_{k}$ is a matrix with size $k \times k$, where only $(k-1, k-1)$ th component is one and other components are zero. For the case of $\hat{B}_{2}^{\text {dir }}$, where dir $\in$ $\{r, l, u, d\}$, the third equations are applied. For the case of $\widehat{B}_{3}^{\mathrm{dir}}$, where $\operatorname{dir} \in\{r, l, u, d\}$, the fourth equations are applied. For the case of $\widehat{B}_{i}^{\mathrm{dir}}$, where $i \in\{1,2,3\}$ and $\operatorname{dir} \in\{r, l, u, d\}$, if the displaced block is fully overlapped with previously obtained MC-DCT blocks, the form of the equation is like the first equations of (13) and (16). However, if the displaced block is partially overlapped with the intersected blocks $A_{i}$, the equation form will be like the first equations of (14) and (15). Therefore, to extract a DCT macroblock displaced by one pixel in any direction from the previously obtained DCT macroblock, the required computation is largely reduced.

\subsection{DCT-domain block matching criterion}

From energy conservation property, the signal energy in the DCT-domain is equal to the energy in the pixel-domain. The base motion vector results in local motion variation in current macroblock. In order to efficiently capture the variation, we define a localized search area. Since the base motion vector $v_{n}^{0}$ is available, block matching between the $(k-1)$ th frame $\left(f_{k-1}\right)$ and the $k$ th frame $\left(f_{k}\right)$ amounts to find the refinement vector $\delta_{n}$ for each target $\hat{\Omega}_{n}$ by MSE. However, DCT-domain MSE is defined by:

$$
\delta_{n}=\arg \left(\min _{\delta \in S_{L}}\left(\sum_{p \in \hat{\Omega}_{n}}\left|\hat{f}_{k}(p)-\hat{f}_{k-1}\left(p+v_{n}^{0}+\delta\right)\right|^{2}\right)\right),
$$

where $\hat{f}_{k}$ is the DCT-domain version of $k$ th frame $f_{k}, \hat{\Omega}_{n}$ is the $n$th DCT macroblock in $\hat{f}_{k}, v_{n}^{0}$ is the base motion vector of $\hat{\Omega}_{n}, p$ is the position vector, $\delta$ is the delta vector, and $S_{L}$ is the local search area (LSA) depending on original motion vectors. The refined motion vector for $\widehat{\Omega}_{n}$ is defined by:

$$
v_{n}=v_{n}^{0}+\delta_{n}
$$

As the nonzero DCT coefficients statistically concentrate on the neighborhood of DC component, only few coefficients are considered in the new criterion. This will alleviate the burden in DCT matching.

\subsection{Fast search (FS) algorithm}

Seo and Kim [9] proposed that base motion vector from the median method is good enough to achieve the small search window $(-2,+2)$. However, it is still not suitable for the MVR in the DCT-domain. Thus, it is highly desired to reduce the search area as much as possible. For this purpose, [9] introduces a localization motion vector (LMV). The LMV is detected by calculating the average of three motion vectors except the base one. Figure 7 shows an example of the proposed fast search algorithm. In this example, the LMV points at $(1,-2)$. The shaded area is called the localized search area, which corresponds to $S_{L}$ in (17). The checkpoints in the localized search area are considered for MVR.

If the LMV points at the vertical or horizontal axes, number of checkpoints is significantly reduced. In this case, a maximum of 3 points are checked for MVR. The example is shown in Figure 7, with 6 points checked. First, $C_{00}$ is checked. $C_{10}$ displaced from $C_{00}$ is checked by using overlapped $16 \times 15$ pixels. Second, $C_{0_{-} 1}$ and $C_{1_{-1}}$ are checked by using the overlapped $15 \times 16$ pixels with the obtained $C_{00}$ and $C_{10}$, respectively. Similarly, $C_{0-2}$ and $C_{1 \_2}$ are checked by using the overlapping information with the obtained $C_{0_{-} 1}$ and $C_{1-1}$, respectively. Through extensive simulations, it was established that if LMV points outside the search window $(-2,+2)$, motion correlation between the four original MVs is low. Thus, MVR effect may be poor or meaningless. In this case, MVR is not performed. Instead, the macroblock type is determined as "INTER_4v." This macroblock type allows four motion vectors for each $8 \times 8$ block forming the $16 \times 16$ macroblock [15]. To generate four motion vectors per macroblock, the incoming motion vectors are scaled down by half to reflect the spatial resolution transcoding. Since the approach 


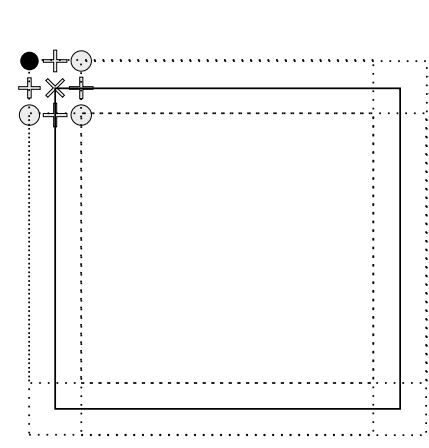

(a)

$\bigcirc$ Integral point

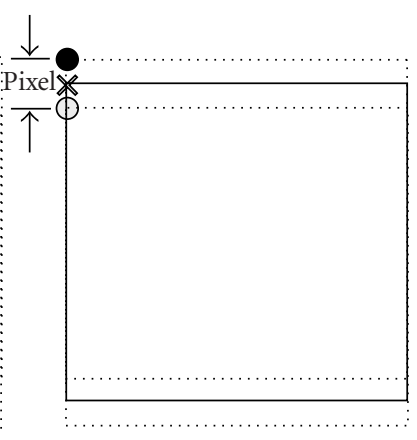

(b)

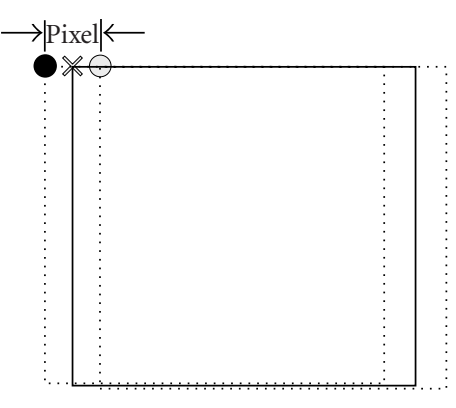

FIgURE 9: (a) $\mathrm{MV}_{x}$ and $\mathrm{MV}_{y}$ are all nonintegral (b) $\mathrm{MV}_{x}$ orMV $y$ is nonintegral.

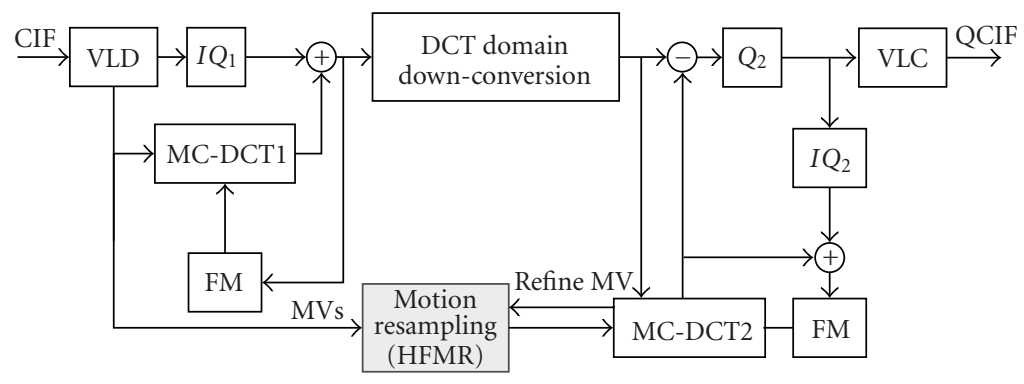

Figure 10: Using FRNI in CDDT.

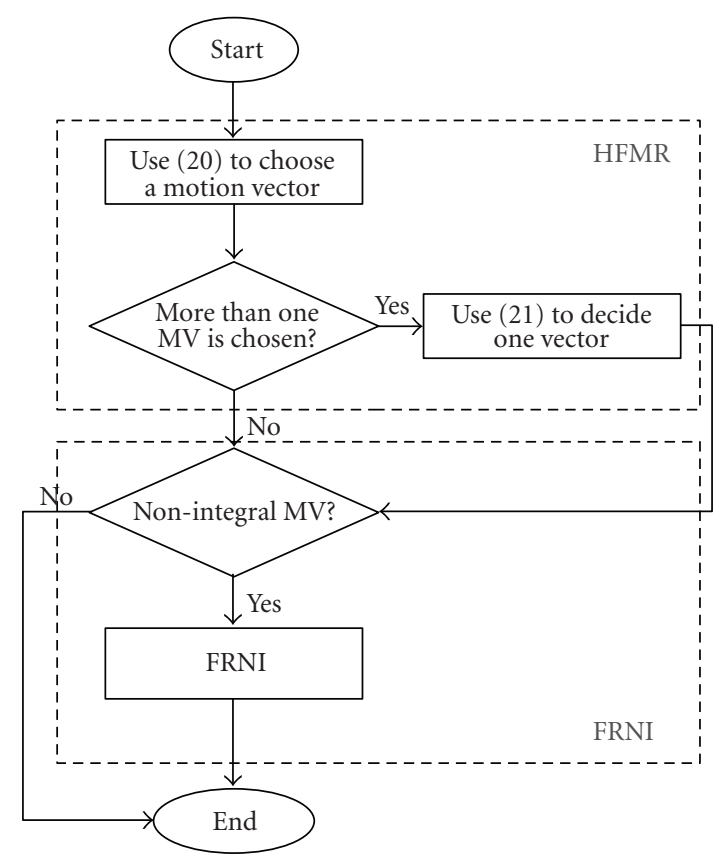

FIgURE 11: Flow chart of the entire proposed HFMR and FRNI.

can apply refinement with fewer point (compared with traditional refinement), it is thereby referred to as fast search (FS).

\section{PROPOSED ARCHITECTURES}

It is obvious that three problems occurred in DCT-domain video transcoding.

(1) It is elaborate to extract macroblock in DCT-domain.

(2) It is difficult to refine motion vector in DCT-domain. Therefore, transcoding in DCT-domain needs more accurate motion vector than pixel domain.

(3) However, nonrefined motion vector is not suitable for video transcoder in DCT-domain.

In order to solve these problems, we propose the following algorithms.

\subsection{Hierarchical fast motion resampling (HFMR)}

Fast motion resampling (FMR) is always performed by simple operations (such as average, median filtering, and weighting) to reduce computation complexity and get new motion vector. It is based on the property of motion vectors and macroblock activity. Among those operations, median filtering can reach general performance for all sequences as in:

$$
v=\frac{1}{2} \arg \min _{\overline{v_{i}} \in\left\{v_{1}, v_{2}, v_{3}, v_{4}\right\}} \sum_{j=1, j \neq i}^{4}\left\|v_{i}-v_{j}\right\| .
$$

However, transcoding in DCT-domain needs more accurate motion vector than pixel-domain and different direction 


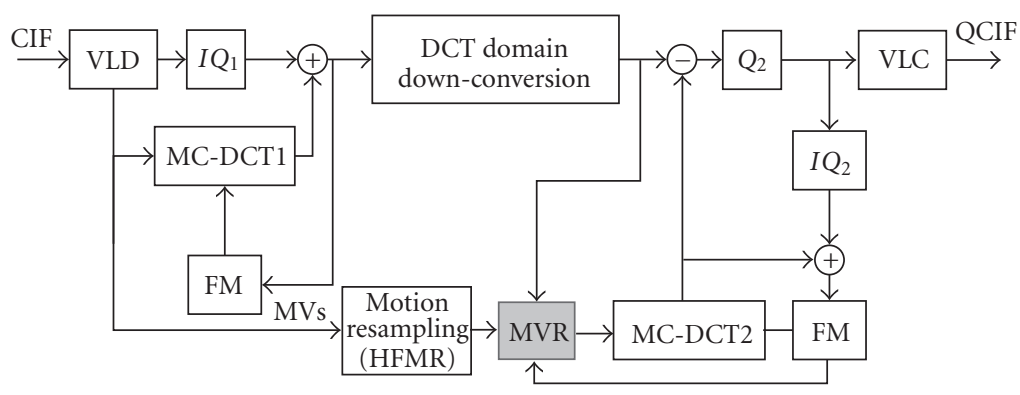

FIgURE 12: The architecture of CDDT with MVR.

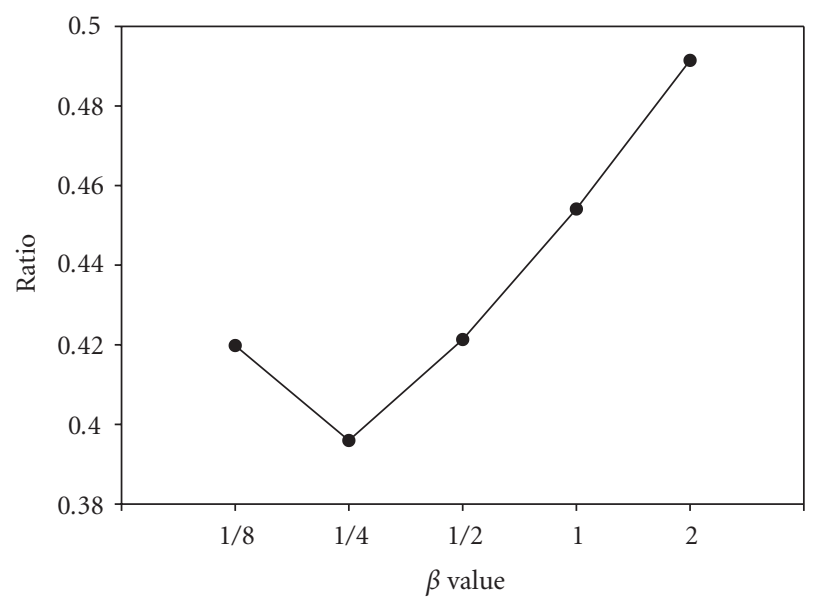

Figure 13: $\beta$ and ratio.

motion vectors have the same value under usual circumstances. For example, considering four motion vectors: $(1,-1),(0,5),(-1,-1)$, and $(0,-5)$, both motion vectors $(1,-1)$ and $(-1,-1)$ have the minimum value as shown in Figure 8 . Therefore, we must further evaluate two motion vectors.

The number of nonzero coefficient is related to the residue energy. When fewer nonzero DCT coefficients are presented, less residue energy indicates that the predicted motion vector is more accurate. According to the observation, the number of nonzero DCT coefficients is applied to decide motion vector in this situation. As in (20), we choose MV by minimum value of $A_{v j}$. However, $v j$ is detected by (19), and all $v j$ are of the equivalent minimum value. $A_{v j}$ denotes the number of nonzero DCT coefficients in macroblock and detected by $v j$ :

$$
v=\frac{1}{2} \arg \min _{\overline{v_{j}} \in\left\{v_{1}, v_{2}, v_{3}, v_{4}\right\}} A_{v j} .
$$

\subsection{Fast refinement for nonintegral MV (FRNI)}

As mentioned above, nonrefined motion vector is not suitable for video transcoder in DCT-domain. However, it is difficult to refine motion vector in DCT-domain. In MCDCT, we have to extract more than one block for nonintegral

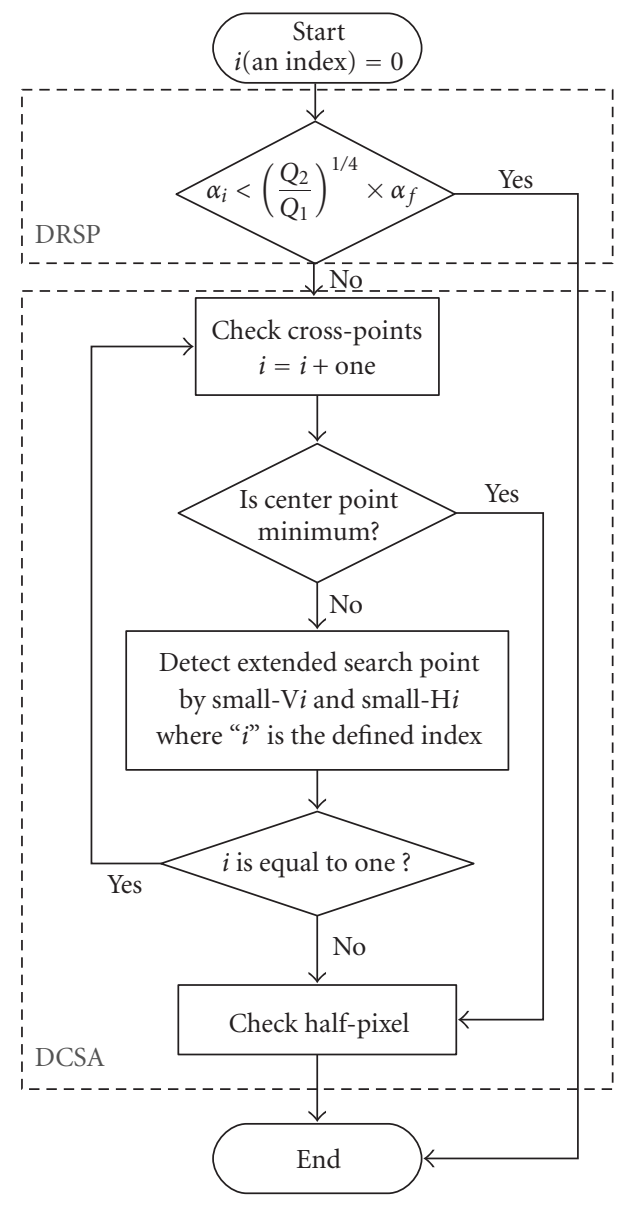

FIGURE 14: Flow chart of the proposed DRS.

motion vector which generated from FMR. As shown in Figure 9, we must extract all blocks of integral point to compose the block of target points which are detected by motion vector. Unfortunately, this motion vector generated by FMR is not always accurate enough.

Therefore, we propose the fast algorithm, FRNI motion vector, to only refine nonintegral motion vector by extracted block. Our proposed FRNI is based on cascaded DCTdomain transcoder (CDDT) shown in Figure 10. The main concept is data reusing in MC-DCT to refine the generated half-pixel motion vector. The difference between the data 


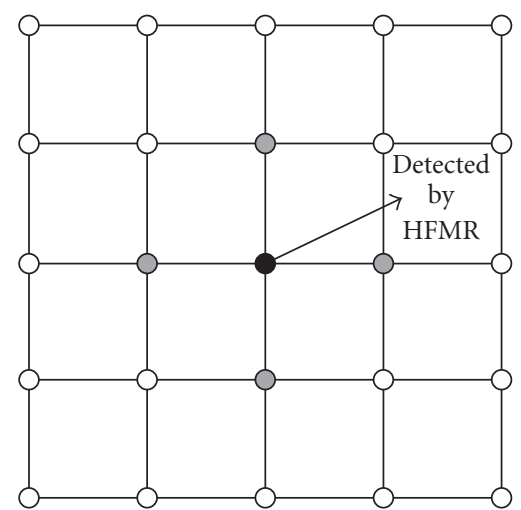

(a)

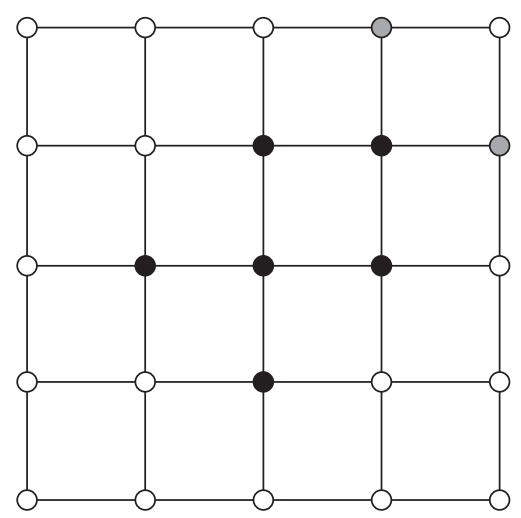

(c)

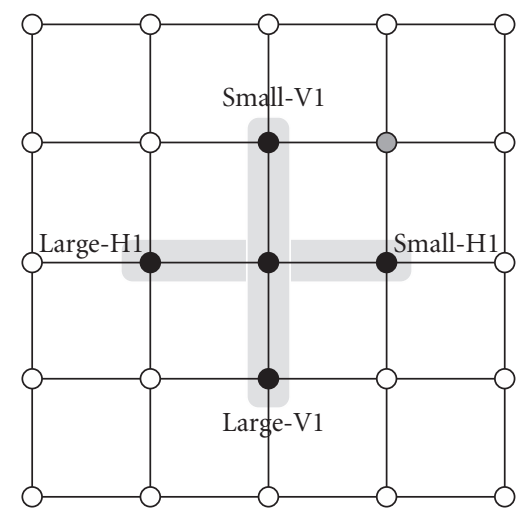

(b)

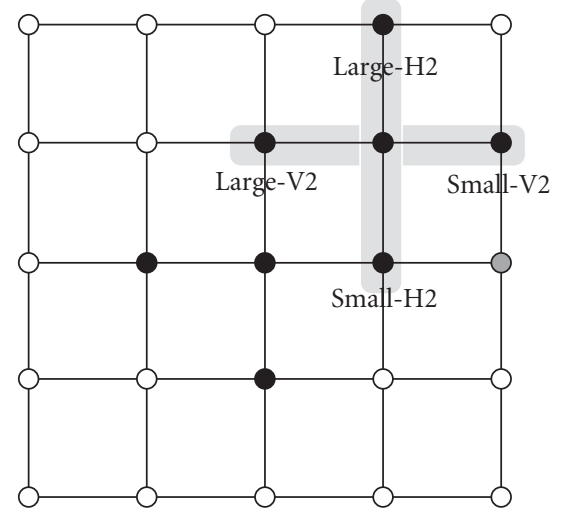

(d)
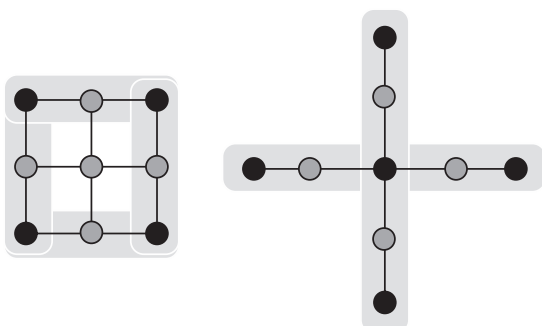

(e)

FIgURE 15: Steps in double cross-search algorithm: (a) Step 1, (b) Step 2, (c) Step 3, (d) Step 4, (e) Step 5 of double cross-search algorithm.

reuse in [9] and the approach is from the fact that the basic motion vector decision, as called resampling, is different.

Due to this concept, we can get more useful data from MC-DCT without paying any additional operation. Furthermore, the proposed algorithm is easily combined with conventional architecture to get a more efficient architecture. Based on our analysis, the computational complexity of extracted DCT block is larger than the criterion function. Therefore, it is essential to utilize the extracted blocks efficiently. As shown in Figure 9, we realize that there are nine additional checkpoints if $\mathrm{MV}_{x}$ and $\mathrm{MV}_{y}$ are all nonintegral in Figure 9(a), and three additional checkpoints if $\mathrm{MV}_{x}$ or $\mathrm{MV}_{y}$ is nonintegral in Figure $9(\mathrm{~b})$. Because the blocks of integral points have been extracted, we can obtain the additional checkpoints directly or by computing the average from extracted blocks. The black point in Figure 9 is decided by rounding nonintegral motion vector. Afterward, we use the absolute sum of DCT coefficients to determine the refined motion vector in (21), where $M V_{\text {offset }}$ is the offset motion vector, $S$ is the checkpoint detected by original motion vector, $\delta$ is the current checkpoint, $\mathrm{MB}_{\delta}$ is the residue block detected by $\delta, a$ is the refinement motion vector distance, and $\mathrm{MB}_{\delta-a}$ is the extracted block from DCT-domain with refined motion vector. The refined $\mathrm{MV}$ is defined as $M V_{\text {Refined }}=M V_{\text {non-refined }}+$ $\mathrm{MV}_{\text {offset: }}$ :

$$
\mathrm{MV}_{\text {offset }}=\arg \min _{\delta \in S} \sum_{a=0}^{3} \sum_{i=0}^{63}\left\|a b s\left(\mathrm{MB}_{\delta-a}\left(\mathrm{DCT}_{i}\right)\right)\right\| .
$$



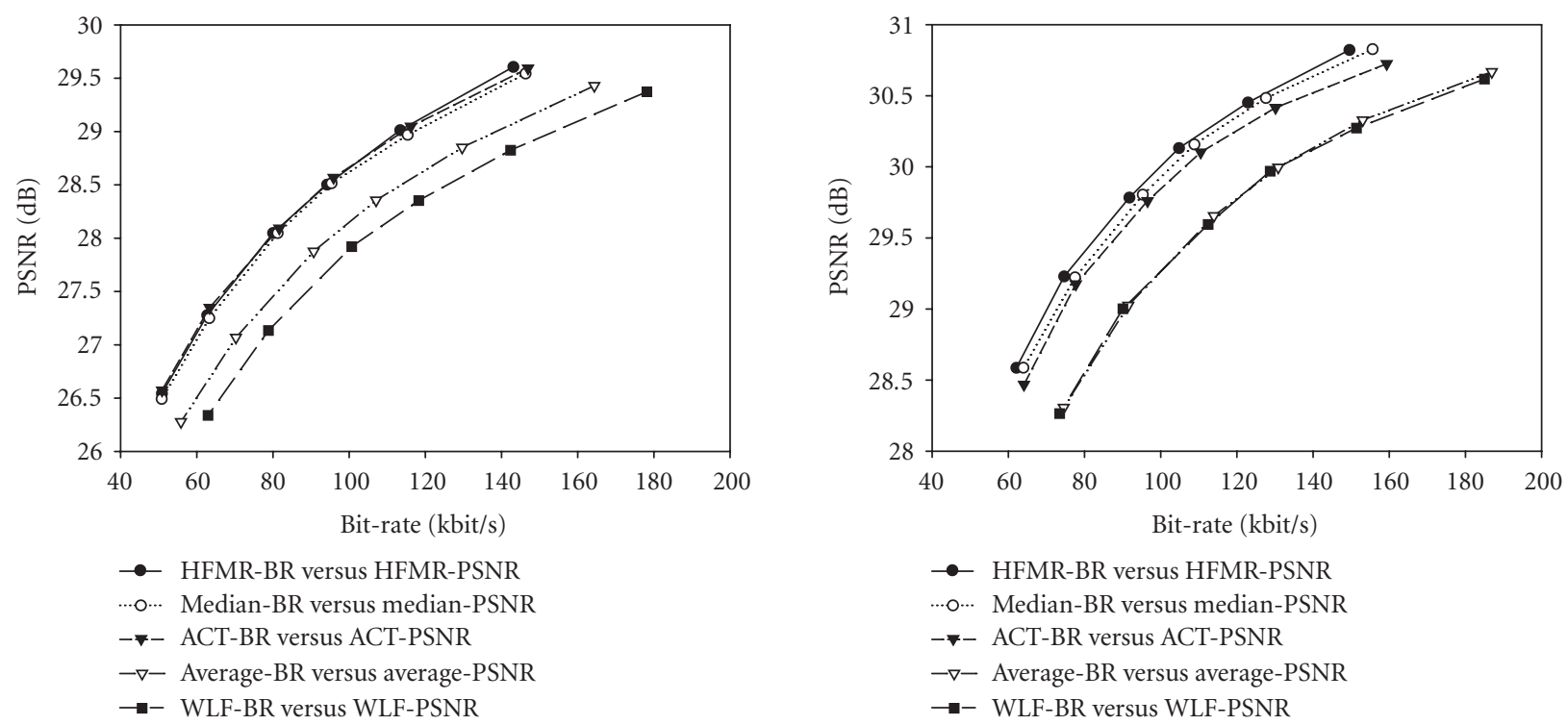

(b)

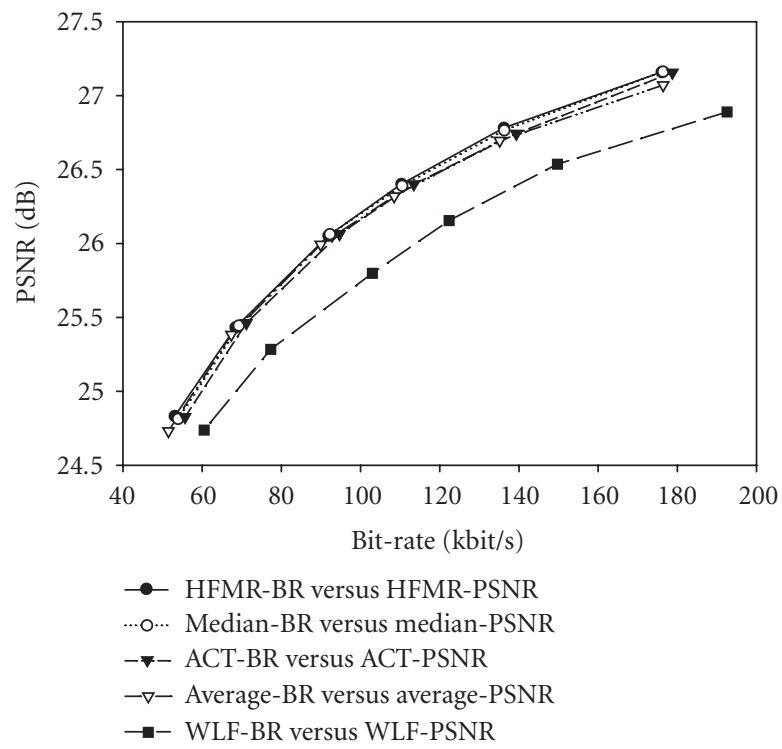

(c)

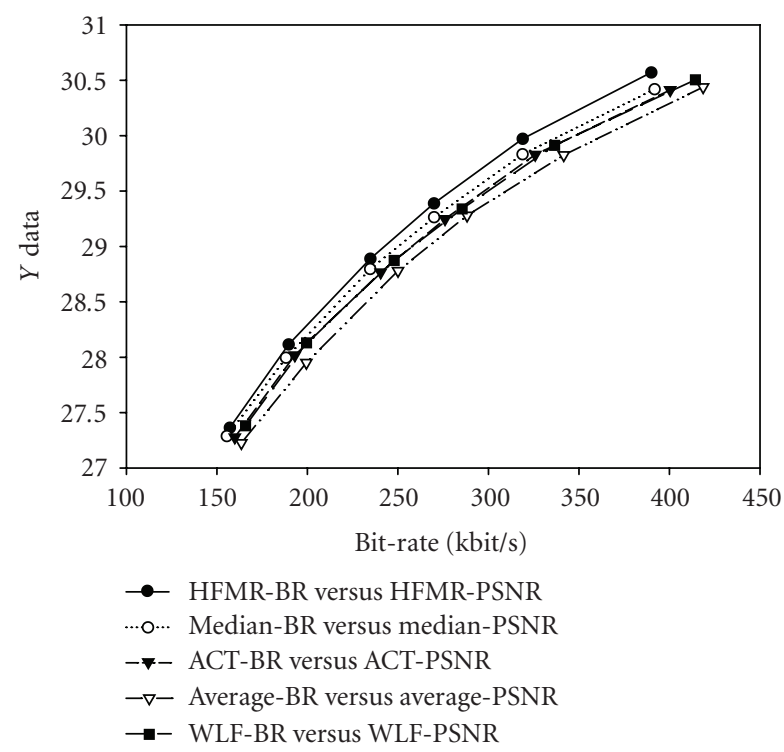

(d)

Figure 16: The R-D curves for different FMR algorithm. (a) Foreman. (b) TableTennis. (c) Coastguard. (d) Football.

By using FRNI, we can get three advantages.

(1) Considering the rate distortion, we can obtain more suitable motion vector.

(2) FRNI does not increase the computation complexity for extracting MBs in DCT-domain.

(3) MV refinement can be separated into integer and half-pixel process. Since half-pixel refinement can be achieved by the integer search with some additions and decisions (based on the definition of FRNI), the complexity on MC-DCT can be reduced. This is obvious in DCT-domain since the compensation and prediction cannot be preformed directly from simple arithmetic.
The flow chart of the entire proposed algorithm is shown in Figure 11. It is constructed by two individual parts. For HFMR, it provides more accurate motion vector. In FRNI, we can get more suitable refined motion vector and reduce complexity of nonintegral motion vector in $\mathrm{MC}$ DCT. Furthermore, the flow chart of the entire proposed algorithm is only performed in luminance component. As chrominance components, the coded type is decided based on its corresponding luminance component.

\subsection{Dynamic regulating search (DRS)}

According to experiment and analysis, the fast search algorithm [9] can control search range in $(-2,2)$ efficiently. However, the complexities of MVR in different bit-streams 


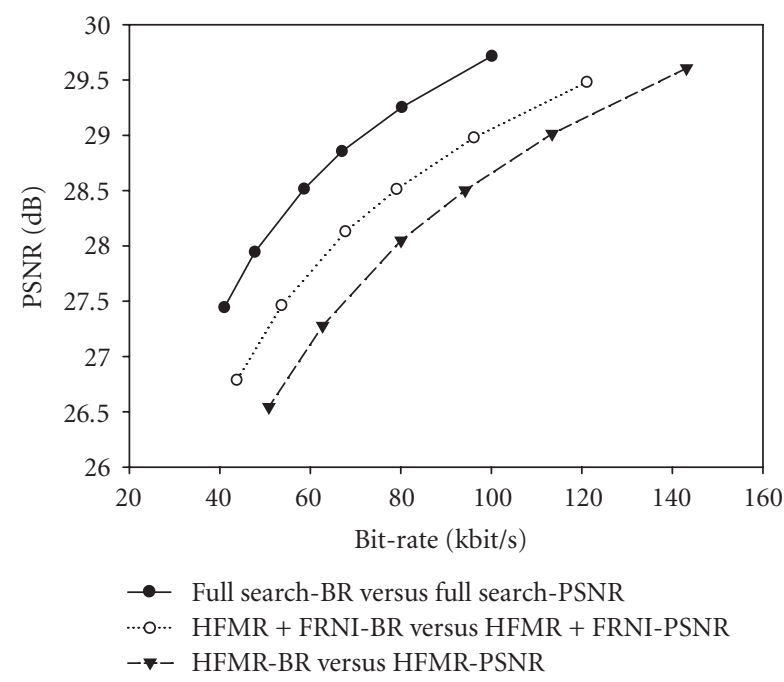

(a)

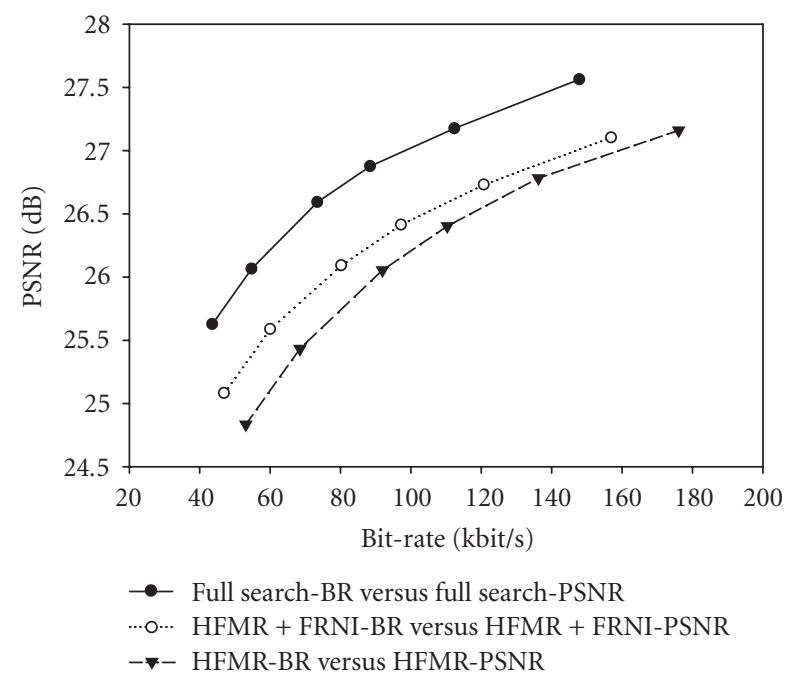

(c)

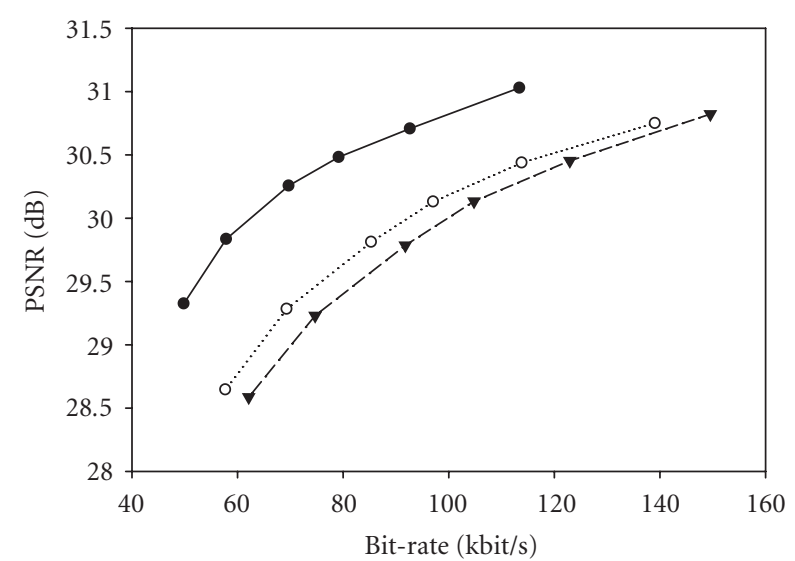

- Full search-BR versus full search-PSNR

… $\quad$ HFMR + FRNI-BR versus HFMR + FRNI-PSNR

- $\mathbf{-}$ - HFMR-BR versus HFMR-PSNR

(b)

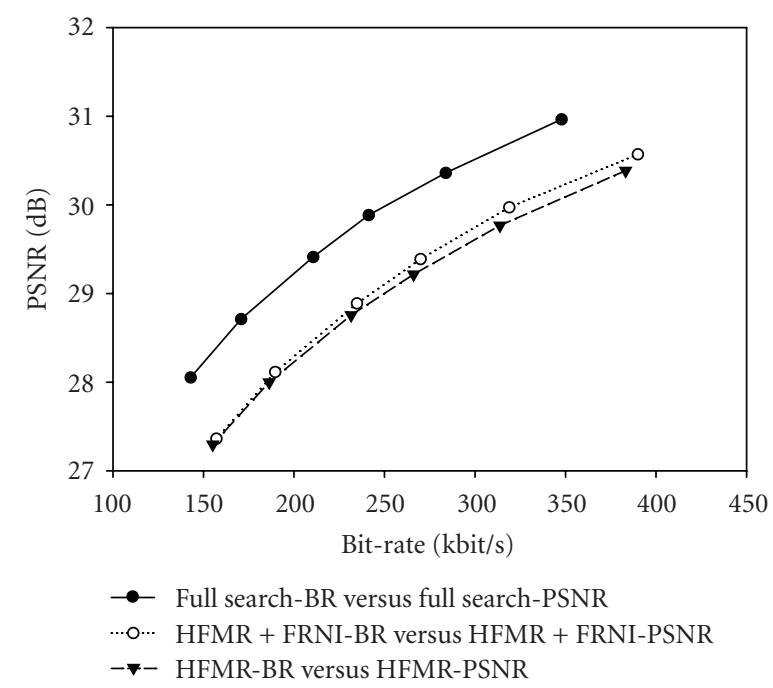

(d)

FIgURe 17: The R-D curves for our proposed FRNI. (a) Foreman. (b) TableTennis. (c) Coastguard. (d) Football.

are the same, since the search range is only decided by four input motion vectors. In video coding technique, quantization error dominates video coding distortions in low bit-stream saturation. Therefore, it is essential to reduce search points dynamically in low bit-rate. Furthermore, we also propose a novel fast MVR algorithm, double crosssearch algorithm (DCSA), to improve the performance of MVR. The proposed algorithms are based on the architecture, as shown in Figure 12.

\subsubsection{Dynamic reduced search points in MVR (DRSP)}

Most of the refinement algorithms try to reduce computational complexity by search range reduction or fast search algorithm. However, there are still heavy computational complexities since all the motion vectors in the frame are re-estimated in the refinement process. Therefore, we utilize the variance of $\alpha$ and $\left(Q_{2} / Q_{1}\right)^{\beta} \times \alpha_{f}$ to be a criterion. $\alpha_{i}$ is the absolute sum of DCT coefficients of current block and $\alpha_{f}$ is the average value of blocks of previous frame. $Q_{1}$ is the quantization parameter of input stream and $Q_{2}$ is the quantization parameter of output stream. $\beta$ is a parameter for refinement threshold definition. To investigate the effect of various $\beta$ on refinement points, an experiment is conducted with 5 patterns $(1 / 8,1 / 4,1 / 2,1,2)$ for $\beta$ and the result is presented in Figure 13. The experiment is performed on various sequences (including Foreman, Costguard, and TableTennis). Since the objective is to minimize refinement point, a criterion function is defined by:

$$
\text { ratio }=\frac{(\text { refined points })_{\beta=0}-(\text { refined points })_{\beta}}{(\text { search point })_{\beta=0}-(\text { search point })_{\beta}} .
$$

The pseudocode of DRSP is described by (23). Based on the simulation result, $1 / 4$ is the most appropriate value for $\beta$. According to the setup for $\beta(1 / 4)$ and $\alpha$, (23) can be 


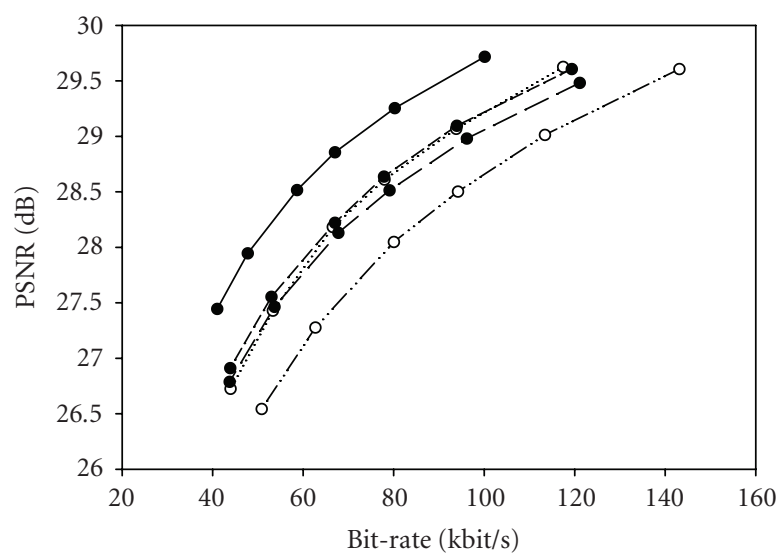

- Full search-BR versus full search-PSNR

...... DRS-BR versus DRS-PSNR

- - FS[9]-BR versus FS[9]-PSNR

-o- HFMR-BR versus HFMR-PSNR

$\rightarrow-$ HFMR + FRNI-BR versus HFMR + FRNI-PSNR

(a)

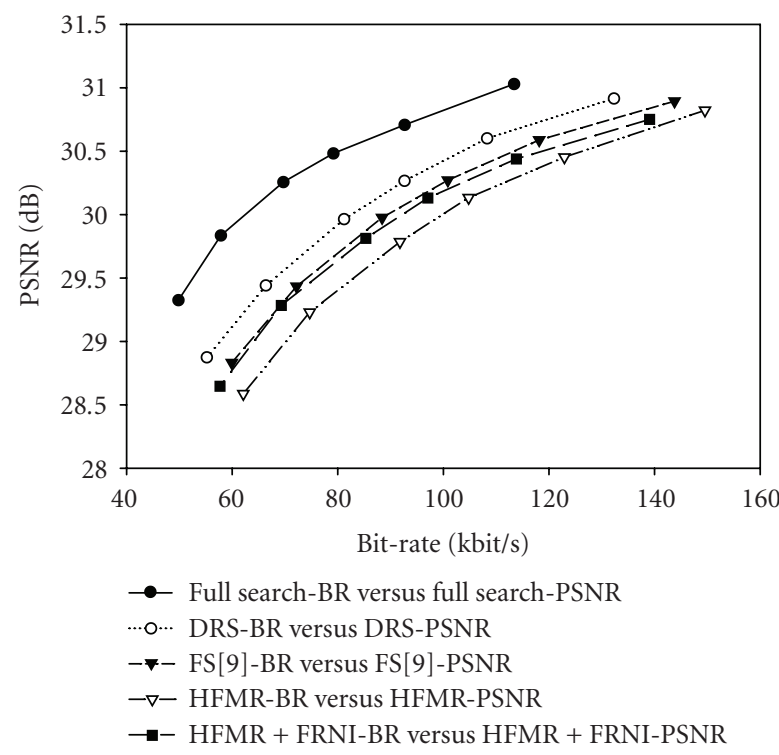

(c)

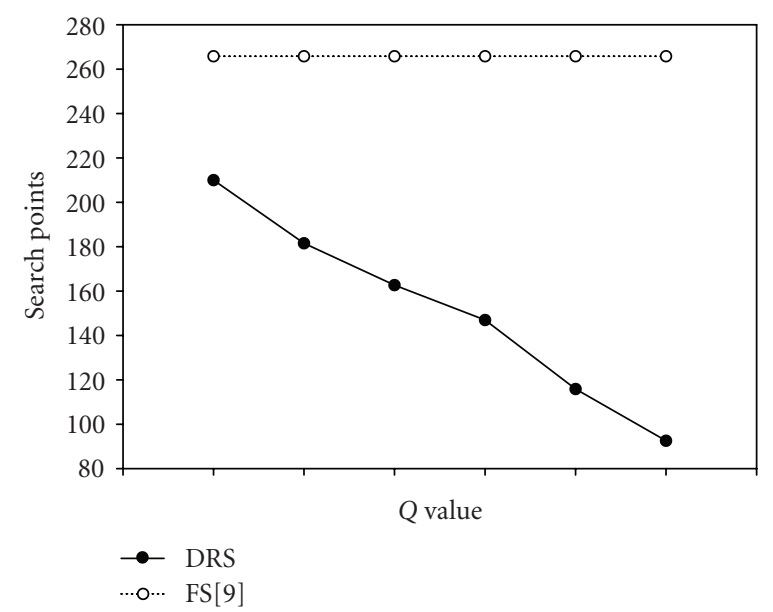

(b)

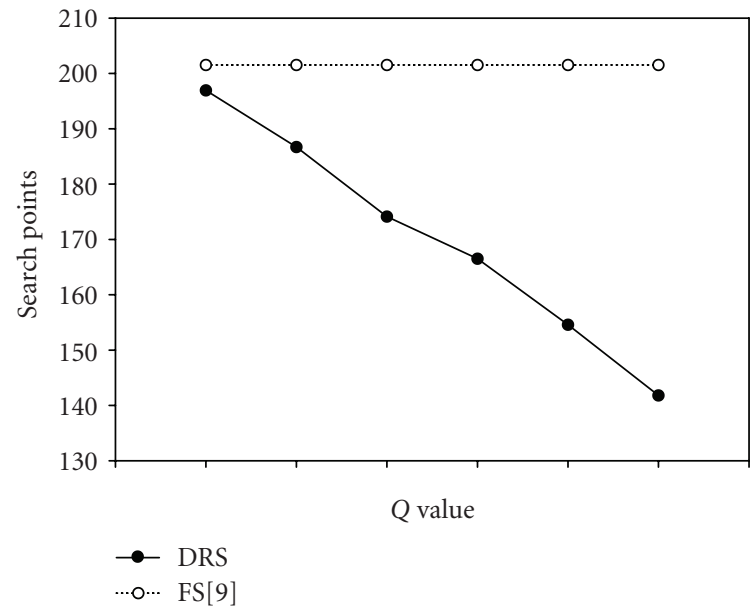

(d)

FIgURE 18: Simulation results for DRS. (a) The R-D curves of Foreman. (b) Average search points per frame for Foreman. (c) The R-D curves of TableTennis. (d) Average search points per frame for TableTennis.

implemented to fulfill the goal in search point reduction:

$$
\text { if }\left(\alpha_{i}<\left(\frac{Q_{1}}{Q_{2}}\right)^{\beta} \times \alpha_{f}\right) \text {, then Non-refinement, }
$$

\subsubsection{Double cross-search algorithm}

After DRSP, we perform double cross-search algorithm for refined blocks. Overall flowchart is presented in Figure 14. When the criterion is matched in DRSP, double cross-search is performed. Initial search is performed on a "cross-like" area, and the shape is demonstrated in Figure 15(a). At the same time, an index " $i$ " is defined and set to one. The index is to number the iteration. If cost function is minimal at the center, refinement is stopped. Else, the search point is expended by the cross shape based on smaller horizontal and vertical motion vector, defined as small-H1 and smallV1. The refinement process repeated if the extension is performed. Since the loop is terminated when index " $i$ " is equal or larger than 2 , maximum iteration number is 2.

The following is an example for the proposed algorithm. Step 1. We use (21) to check the 4-neighboring points (up, down, left, and right). If the checkpoints are smaller than the center point, we expand search point in Step 2; otherwise, stop the search (Step 5). The process is shown in Figure 15(a). 


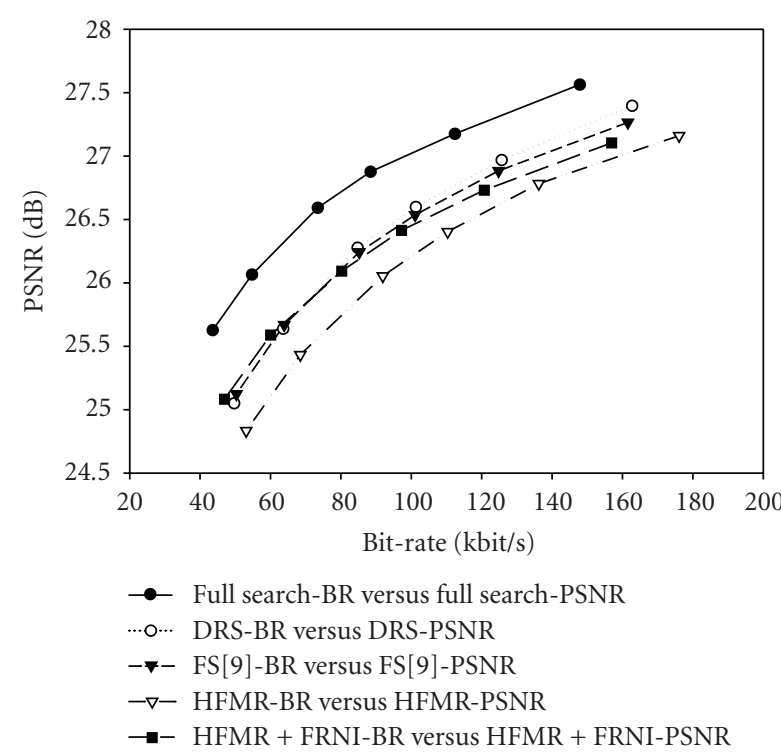

(a)

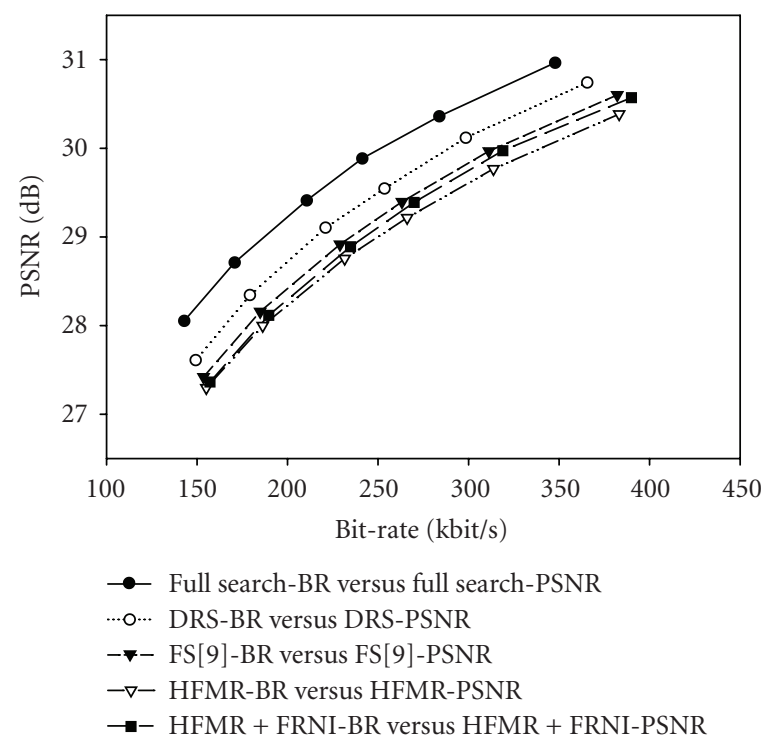

(c)

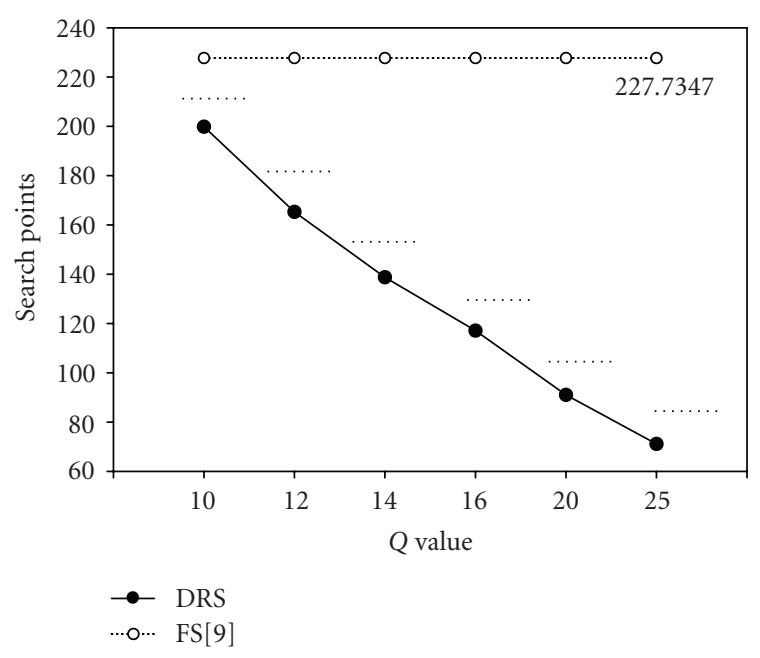

(b)

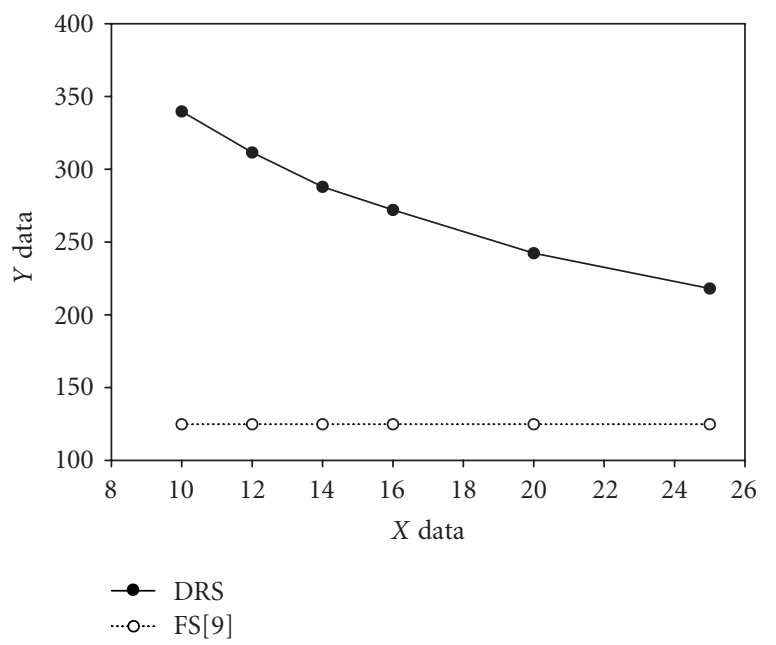

(d)

FIGURE 19: Simulation results for DRS. (a) The R-D curves of Coastguard. (b) Average search points per frame for Coastguard. (c) The R-D curves of Football. (d) Average search points per frame for Football.

Step 2. The expanded point is decided by Small-V1 and Small-H1. If the expanded point is smaller than Small-V1 and Small-H1, we expand search points in Step 3 further; otherwise, stop the search (Step 5). The process is as shown in Figure 15(b).

Step 3. As shown in Figure 15(c), we expand horizontal and vertical search points. If the prediction error of any checkpoint is smaller than the center one, we expand search point in Step 4; otherwise, stop search (Step 5).

Step 4. The expanded point is decided by Small-V2 and Small-H2, as shown in Figure 15(d).

Step 5. We perform search in half-pixel according to final search point. As shown in Figure 15(e), there are two possible patterns.
In addition to improving the efficiency of search, the proposed DRS has several advantages. First, we can control search range in $(-2,2)$ efficiently in Step 2 . Second, we can search half-pixel points efficiently. Finally, we can reduce complexity further by combining with the efficient method in [9] for block extracting.

\subsection{Overall design}

In previous subsections, techniques are proposed including the HFMR with accuracy motion resampling, the FRNI for MV, and the DRS with low-complexity MVR. Two kinds of MVR algorithms are designed for different architectures. Based on brute-force MC-DCT, FRNI can provide better 
TABLE 3: Experimental results for three simulated sequences at $\mathrm{QP}=10$.

\begin{tabular}{lccc}
\hline Sequence name & FMR & Bitrate & PSNR \\
\hline \multirow{3}{*}{ Foreman } & HFMR & 143.12 & 29.6063 \\
& Median & 146.31 & 29.5446 \\
& ACT-weighted & 146.95 & 29.5928 \\
& Average & 164.38 & 29.4295 \\
& WLF & 178.17 & 29.3728 \\
\hline \multirow{3}{*}{ TableTennis } & HFMR & 149.55 & 30.822 \\
& Median & 155.63 & 30.8285 \\
& ACT-weighted & 159.34 & 30.7248 \\
& Average & 186.92 & 30.668 \\
Coastguard & WLF & 185 & 30.6177 \\
& HFMR & 176.09 & 27.1596 \\
& Median & 176.41 & 27.1623 \\
& ACT-weighted & 178.77 & 27.1558 \\
& Average & 176.42 & 27.0714 \\
& WLF & 192.57 & 26.8894 \\
\hline
\end{tabular}

TABLE 4: Reduced ratio of nonintegral in MC-DCT.

\begin{tabular}{llcc}
\hline Sequence name & $\begin{array}{c}\text { Number of extracted DCT blocks for nonintegral MV (4 luminance blocks and 2 chrominance blocks) } \\
\text { Nonrefined }\end{array}$ & Reduced ratio \\
\hline Foreman & $4 \times 6544+2 \times 9114$ & $4 \times 6544+2 \times 7220$ & $8.50 \%$ \\
Table & $4 \times 6560+2 \times 8976$ & $4 \times 6560+2 \times 6696$ & $11.30 \%$ \\
Coastguard & $4 \times 6100+2 \times 7904$ & $4 \times 6100+2 \times 6738$ & $5.8 \%$ \\
\hline
\end{tabular}

quality than nonrefine motion vector and reduce the complexity. DRS can utilize the filter for half-pixel motion vector in MC-DCT [8] and efficient method for extracting MCDCT block [9] to improve the performance.

\section{EXPERIMENTAL RESULTS}

\subsection{Experiment environment}

We simulate the proposed algorithms in software by transcoding H.263 CIF $(352 \times 288)$ bit streams to QCIF (176 by 144). The simulated four well-known sequences are Foreman, Coastguard, TableTennis, and Football. Foreman and TableTennis is with median motion activity. Costguard is with low motion activity. Football is with high motion activity. Since the performance is deeply related with motion activity, the sequences can show the performance under various circumstances. In order to get better comparison with the other methods, the coding style of each input stream is IPPPPPP with 50 frames and 30 frames/s. The test sequences are first encoded at quantization parameter $(\mathrm{QP})$ of 6. Afterward, six transcoders (with $\mathrm{QP}=10,12,14,16$, 20 , and 25) are applied for transcoding, respectively. The full search range is $(-2,2)$.

\subsection{Experiment result of HFMR}

We compare with the other FMR algorithms, ACT-weight, median filter, average, and WLF-max. The rate-distortion (RD) curves of test sequences, Foreman, TableTennis, Costguard, and Football, are shown in Figures 16(a), 16(b), 16(c), and 16(d), respectively. In Table 3, we also show the average PSNR and bit-rate at $\mathrm{QP}=10$ for three sequences. The objective of HFMR is to ensure consistent quality among various video sequences. Thus, slight modification is made on conventional approaches. According to the experimental results, HFMR is with highest quality among all. The reason for similar performance is from the fact that resampling applies simple method to obtain a rough motion vector. Since it is not refined extensively, the effect on performance is limited.

\subsection{Experiment result of FRNI}

In this subsection, we show the experiment results of FRNI for the test sequences. It is quite clear that the performance for all test sequences (Foreman, TableTennis, Coastguard, and Football) is improved at various QPs after combining with FRNI shown in Figures 17(a), 17(b), 17(c), and 17(d), respectively.

In Table 4, we show the total numbers of extracted DCT blocks for half-pixel motion vectors and the reduced ratios. The results prove that the proposed FRNI will not induce extra computational complexity in MC-DCT. However, it can get about $8.5 \%$ of reduction with the proposed HFMR in average. Note that the reduced operation of extracted block is larger than the checked point.

\subsection{Experiment result of DRS}

The experiment results of DRS for four test sequences are shown in Figures 18, 19(a), and 19(c). The comparisons of 
search points between [9] and DRS are shown in Figures 18, and 19(b), and 19(d). Quantization parameters for (a)-(c) and (b)-(d) are the same, as illustrated in (b)-(d). For the best case (TableTennis) DRS can improve performance and reduce search points in all different QPs. The reduction of search points is about 29\%, especially at QP of 25 . As to the normal cases, we do not only preserve the quality but also reduce $21 \% \sim 65 \%$ and $12 \% \sim 69 \%$ of search points for Forman and Coastguard, respectively. The only exception falls in the sequence Football. However, the performance of DRS is obviously higher than FS. From the results, proposed DRS can achieve higher performance (compared with FS) while the search point is reduced in average. When the search point is higher, performance of DRS is relatively strong compared with other algorithms.

The search point for HFMR-FRNI is smaller than DRS. However, the performance in DRS is better than HFMRFRNI. Since it is a tradeoff between search point and performance, the adoption of specific approach is considered based on the given constraint. If faster approach is desired, HFMR-FRNI is a better choice. If better performance is desired, DRS is the best candidate.

\section{CONCLUSION}

This article presented several techniques for spatial resolution reduction for video transcoder in DCT-domain. For motion vector resampling, our proposed HFMR modified the median filter to improve the entire quality further. As to MVR, we proposed FRNI and DRS for different architectures. FRNI is based on brute-force MC-DCT to only refine half-pixel motion vector. DRS reduced search points dynamically and achieved efficient refinement by DRSP and DCSA, respectively.

From the experiments, we believe that the proposed HFMR outperforms the other techniques for all simulated sequences. Besides, two MVR algorithms also improved the entire quality efficiently, especially DRS. For the best case, DRS can improve performance and reduce search points in all different QPs. Compared with [9], the reduction of search points is about $29 \%$. As to the normal cases, it does not only preserved the quality but also reduced $16 \% \sim 67 \%$ of search points.

\section{REFERENCES}

[1] A. Vetro, C. Christopoulos, and H. Sun, "Video transcoding architectures and techniques: an overview," IEEE Signal Processing Magazine, vol. 20, no. 2, pp. 18-29, 2003.

[2] P. Yin, A. Vetro, B. Liu, and H. Sun, "Drift compensation for reduced spatial resolution transcoding," IEEE Transactions on Circuits and Systems for Video Technology, vol. 12, no. 11, pp. 1009-1020, 2002.

[3] W. Zhu, K. H. Yang, and M. J. Beacken, "CIF-to-QCIF video bitstream down-conversion in the DCT domain," Bell Labs Technical Journal, vol. 3, no. 3, pp. 21-29, 1998.

[4] B. Shen, I. K. Sethi, and B. Vasudev, "Adaptive motionvector resampling for compressed video downscaling," IEEE Transactions on Circuits and Systems for Video Technology, vol. 9, no. 6, pp. 929-936, 1999.
[5] K.-T. Fung and W.-C. Siu, "DCT-based video downscaling transcoder using split and merge technique," IEEE Transactions on Image Processing, vol. 15, no. 2, pp. 394-403, 2006.

[6] Y.-R. Lee and C.-W. Lin, "DCT-domain spatial transcoding using generalized DCT decimation," in Proceedings of IEEE International Conference on Image Processing (ICIP '05), vol. 1, pp. 821-824, Genova, Italy, September 2005.

[7] Y.-R. Lee and C.-W. Lin, "Visual quality enhancement in DCTdomain spatial downscaling transcoding using generalized DCT decimation," IEEE Transactions on Circuits and Systems for Video Technology, vol. 17, no. 8, pp. 1079-1084, 2007.

[8] G. Cao, Z. Lei, J. Li, N. D. Georganas, and Z. Zhu, "A novel DCT domain transcoder for transcoding video streams with half-pixel motion vectors," Real-Time Imaging, vol. 10, no. 5, pp. 331-337, 2004.

[9] K.-D. Seo and J.-K. Kim, "Fast motion vector re-estimation for transcoding MPEG-1 into MPEG-4 with lower spatial resolution in DCT-domain," Signal Processing: Image Communication, vol. 19, no. 4, pp. 299-312, 2004.

[10] S.-F. Chang and D. G. Messerschmitt, "A new approach to decoding and compositing motion-compensated DCT-based images," in Proceedings of IEEE International Conference on Acoustics, Speech and Signal Processing (ICASSP '93), vol. 5, pp. 421-424, Minneapolis, Minn, USA, April 1993.

[11] N. Merhav and V. Bhaskaran, "Fast algorithms for DCTdomain image downsampling and for inverse motion compensation," IEEE Transactions on Circuits and Systems for Video Technology, vol. 7, no. 3, pp. 468-476, 1997.

[12] N. Merhav, "Multiplication-free approximate algorithms for compressed-domain linear operations on images," IEEE Transactions on Image Processing, vol. 8, no. 2, pp. 247-254, 1999.

[13] K. N. Ngan, D. Chai, and A. Millin, "Very low bit rate video coding using H.263 coder," IEEE Transactions on Circuits and Systems for Video Technology, vol. 6, no. 3, pp. 308-312, 1996.

[14] Y.-R. Lee, C.-W. Lin, and C.-C. Kao, "A DCT-domain video transcoder for spatial resolution downconversion," in Proceedings of the 5th International Conference on Recent Advances in Visual Information Systems (VISUAL '02), vol. 2314 of Lecture Notes in Computer Science, pp. 207-218, Hsin Chu, Taiwan, March 2002.

[15] A. K. Jain, Fundamentals of Digital Image Processing, Prentice Hall, Englewood Cliffs, NJ, USA, 1989. 